\title{
Linx
}

Revue des linguistes de l'université Paris X Nanterre

$55 \mid 2006$

Thème et thématisation

\section{Thématisation, ruptures thématiques et réécriture dans les écrits scolaires}

Marie-Laure Elalouf et Carole Tisset

\section{(2) OpenEdition}

\section{Journals}

Édition électronique

URL : http://journals.openedition.org/linx/398

DOI : $10.4000 /$ linx.398

ISSN : 2118-9692

Éditeur

Presses universitaires de Paris Nanterre

\section{Édition imprimée}

Date de publication : 1 décembre 2006

Pagination : 91-112

ISSN : 0246-8743

\section{Référence électronique}

Marie-Laure Elalouf et Carole Tisset, «Thématisation, ruptures thématiques et réécriture dans les

écrits scolaires », Linx [En ligne], 55 | 2006, mis en ligne le 23 février 2011, consulté le 19 avril 2019.

URL : http://journals.openedition.org/linx/398 ; DOI : 10.4000/linx.398 


\title{
Thématisation, ruptures thématiques et réécriture dans les écrits scolaires
}

\author{
Marie-Laure Elalouf - UMR 7114 Modyco Paris X-CNRS \\ Carole Tisset - UMR 8127 METADIF \\ ml.elalouf@wanadoo.fr
}

Longtemps, les élèves ont appris à écrire des phrases courtes et bien construites, avant d'aborder la rédaction qui prônait les mêmes exigences normatives; il en résultait dans bien des cas une grande pauvreté narrative. Depuis les années 1980, les enseignants de l'école élémentaire sont incités à faire écrire des textes longs, de genres différents (Schneuwly, 1997), sous forme de projet si possible, afin que les élèves produisent de vrais textes et non des suites de phrases. Cette orientation a pour fondements théoriques les travaux de typologie textuelle (Adam, 1984), les recherches sur le fonctionnement des discours (Bronckart, 1985) et la didactique de l'écriture (Garcia-Debanc, 1986, David \& Plane, 1996). Faire écrire aux élèves de « vrais » textes expose toutefois à de nouvelles difficultés très tôt identifiées (Combettes, 1978) et théorisées (Combettes, 1988, Charolles, 1989) : elles relèvent de la cohérence textuelle qui passe par différentes formes de thématisation ${ }^{1}$.

Or, la thématisation n'est pas l'objet d'un apprentissage à l'école élémentaire. Les nouveaux programmes du cycle 3 de l'école primaire enregistrent cette difficulté des élèves à gérer des progressions complexes. On peut lire, dans la section « observation réfléchie de la langue », ce constat suivi d'une recommandation :

\footnotetext{
${ }^{1}$ Sur la notion même de thématisation, Cf. Danes, F., 1974 et Mathesius, V., 1975.
} 
Marie-Laure Elalouf et Carole Tisset

La fermeté du choix énonciatif présidant à la production d'un texte est une conquête difficile pour les élèves de l'école primaire et reste à cet âge plus implicite que réfléchie. Au cycle 3, il suffit de rendre les élèves sensibles aux ruptures qui surviennent dans leurs productions et de les conduire à rétablir l'homogénéité, en particulier dans les textes narratifs, mais aussi dans les projets d'écriture qui interviennent dans d'autres domaines.

Dans les programmes, ruptures a un sens exclusivement négatif. Il suffirait de rétablir une continuité pour que les textes des élèves deviennent satisfaisants. Ce présupposé élude une vraie difficulté : tout texte comporte des ruptures puisque sa cohérence repose sur une tension entre l'introduction d'éléments nouveaux et la reprise d'éléments connus. Ces ruptures peuvent avoir deux origines. Elles peuvent naître d'une discontinuité résultant de la difficulté à gérer les différents états d'un même texte. La génétique textuelle s'est d'ailleurs intéressée aux brouillons d'élèves (Fabre-Cols, 2000 ; pour un état de la question : Anis \& Boré, 2004), de même que la psycholinguistique (Fayol \& Gombert, 1987). Ces discontinuités peuvent aussi provenir d'une mauvaise gestion de la progression d'un texte, du passage d'un type de progression à un autre. Comment gérer ces ruptures pour qu'elles soient perçues comme un élément de progression et non un obstacle à la communication ? Cette question est rarement posée.

En formation initiale, il est d'usage de présenter les différentes progressions thématiques telles que les a analysées B. Combettes (1983) afin que les étudiants soient sensibles aux difficultés de cohésion textuelle dans les textes d'élèves. On invite régulièrement les futurs enseignants à analyser des productions écrites dont la continuité thématique est mal assurée, mais l'exercice n'entre guère dans la pratique malgré l'existence d'ouvrages de référence (Reichler-Béguelin \& al., 1988). Presque aucun manuel, par exemple, ne propose de travailler explicitement sur la thématisation ${ }^{2}$. Pourtant l'activité la plus fréquente, en production de texte, est l'écriture d'une suite narrative, ce qui exige une progression thématique adaptée aux contraintes du texte inducteur. Les ruptures thématiques sont passées sous silence et ne font pas l'objet d'un enseignement précis, sans doute, parce que l'école n'aime pas montrer des erreurs ou des textes qui dysfonctionnent. Les enseignants relèvent ces "incohérences", signalent les discontinuités dans les chaînes anaphoriques des personnages, mais ils restent démunis pour traiter ces questions. En effet, la lecture des écrits scolaires demande un apprentissage spécifique. De nombreuses publications y sont consacrées mais pénètrent insuffisamment la formation des enseignants (Bautier \& Bucheton, 1995 ; Bonnet, Corblin \& Elalouf, 1998 ; Halté, J.-F., Petitjean, A., Plane, S., 2002 ; Plane, 2002-03).

\footnotetext{
${ }^{2}$ Seul le manuel de R.Tomassonne et C.Leu-Simon, Grammaire pour lire et pour écrire (Delagrave, 1997) y consacre un chapitre (p.42-45). Néanmoins, les enseignants du primaire qui utilisent ce manuel font-ils un pont entre cette « leçon » et les ruptures textuelles produites par leurs élèves ?
} 
Au collège, les notions de linguistique textuelle figurent au programme du cycle central $\left(5^{\mathrm{e}}-4^{\mathrm{e}}\right)$ : « thème et propos », «formes simples de thématisation dans le texte (à thème constant, linéaire, éclaté ", "formes simples de thématisation (mise en relief, voix passive) ». Les manuels proposent des repérages sur de courtes séquences homogènes en lecture, mais il est rare que ces notions soient convoquées dans l'écriture ou la réécriture. Les questions posées par l'introduction d'éléments nouveaux (Combettes, Tomassone, 1991) ne sont pas abordées. Derrière cette frilosité, on peut lire un débat sur le rôle des modèles issus de la linguistique textuelle en didactique (Garcia-Debanc, Confais \& Grandaty, 2001) : trop souvent compris comme des modèles à imiter, ils perdent leur statut d'outil heuristique partiel, conçu pour interroger la complexité et non la simplifier. Ainsi, les différents types de progression sont-ils présentés pour euxmêmes, dans des exemples prototypiques, sans devenir des outils pour penser l'hétérogénéité textuelle.

Les dispositifs d'écriture présentés ici s'écartent des pratiques dominantes en ceci : au lieu de postuler une illusoire continuité thématique, ils sont conçus pour provoquer des ruptures et les travailler, en obligeant les élèves à user de progressions auxquelles ils n'auraient pas spontanément recours et en ménageant des temps de réécriture permettant aux écrits d'évoluer. Ils transforment ainsi les difficultés occultées en problèmes à résoudre et invitent les élèves à explorer les potentialités de la langue.

On peut considérer en effet qu'à la fin de l'école primaire, les élèves maitrisent dans leurs écrits la progression à thème constant centrée sur un objet de discours mais rencontrent des difficultés dès qu'il s'agit d'adapter l'enchainement thème-rhème à des besoins de communication qui exigent d'adopter une progression plus complexe ou de marquer des ruptures thématiques, par exemple pour introduire un objet de discours complètement nouveau (De Weck, 1991). Or, comme le rappellent B. Combettes et R. Tomassone (1991), « un élément 'nouveau', auquel on donne une valeur thématique [dans la mesure où il est le point de départ de l'énoncé] peut fort bien être introduit sans présentatif, sans lien avec le contexte ». Dans les textes qu'ils lisent, les élèves rencontrent de telles ruptures, sans forcément être sensibles à ce qui les rend possibles : des réseaux lexicaux et anaphoriques, parfois renforcés par des parallélismes syntaxiques, permettent généralement à l'élément nouveau d'être mis en relief tout en s'inscrivant dans un contexte, comme dans cet extrait d'un roman de littérature de jeunesse lu par la classe de $5^{\mathrm{e}}$ dont nous étudierons certains écrits :

La taverne est sombre et remplie d'hommes assis sur les joncs qui recouvrent la terre battue. Dans les coins les plus obscurs, des chandelles sont allumées. Une forte odeur de bière et de poisson emplit la salle. Un chien efflanqué erre entre les convives. (Odile Weulersse, Le Chevalier au bouclier vert, Hachette jeunesse, pp. 154 à 155)

Confrontés à un problème d'écriture du même ordre, les élèves vont devoir mobiliser des ressources linguistiques, développer des compétences de relecture et de réécriture. Nous allons présenter deux dispositifs d'écriture, l'un en CM2, l'autre en 5 e, qui illustrent cette démarche et analyser les écrits des élèves dans leurs différents états 
de façon à mettre en évidence les ruptures thématiques dues à la consigne d'écriture, en distinguant celles qui perturbent la cohésion textuelle et celles qui instaurent une autre forme de cohésion.

\section{Un dispositif d'écriture en début de CM2}

L'enseignante fait travailler la production d'écrits par jets successifs afin d'obtenir une certaine amélioration des textes produits. De plus, elle y consacre un temps important. Les élèves écrivent régulièrement tous les mardis et tous les jeudis parce que pour elle, la régularité, la quantité et la diversité sont les garants d'une meilleure maitrise.

\section{1- Situation de la production d'écrits dans la progression annuelle}

L'enseignante de ce CM2, un peu désespérée par la pauvreté des écrits constatée en début d'année, a décidé de commencer par des productions sous forme de liste énumérative : j'aime/ je n'aime pas ou les petits riens qui ensoleillent la vie. Puis, la troisième semaine, elle tente de faire passer les élèves de la liste au texte. Elle choisit donc délibérément une difficulté de cohérence textuelle, un «tas de phrases ne form[ant] pas un texte », comme le dit R. Tomassone (op. cit., note 2).

La maîtresse emmène les élèves observer et prendre des notes dans la cour de récréation afin qu'ils produisent un inventaire sous la forme de GN décrivant la cour. Elle propose une piste centrée sur le sujet percevant grâce à l'utilisation de verbes de sensation qu'elle offre puisque la consigne est de «noter ce qu'on voit, ce qu'on entend, ce qu'on sent». Revenus en classe, à partir des notes, les élèves doivent rédiger un texte descriptif sans que le terme "description » ne soit prononcé. L’hyperthème est donné par le titre écrit au tableau :

Dans la cour de récréation, après la récréation du matin

La transformation des notes en texte apparaît dans les aides apportées par l'enseignante. Au cours de la phase de rédaction, l'enseignante intervient oralement et exige :

- que le texte ne comporte plus de tirets ou de numéros comme sur une liste ;

- qu'il soit constitué d'une série de phrases, c'est-à-dire en termes de norme scolaire de phrases ponctuées possédant un verbe conjugué ;

- que la répétition des ily a, on voit soit supprimée.

Puis, elle montre en oral collectif des opérations de reformulation où l'on perçoit ses propres modèles littéraires. À partir d'une phrase d'élève: "je vois des voitures », elle propose de prendre «voitures» comme sujet et de trouver un verbe qui conviendrait. Les élèves proposent: «dessus (le parking), des voitures sont garées». Cette transformation suppose que les élèves fassent une progression à thèmes dérivés en déclinant chaque constituant de la cour, chaque objet discursif. Mais cette reformulation entraîne des ruptures thématiques. Nous allons illustrer ce problème par trois cas. 


\subsection{Ruptures thématiques induites par la contrainte textuelle exigée par l'enseignant}

Le premier cas rend patente la difficulté des élèves à passer d'une progression à thème constant centrée sur la personne ou le personnage de leur texte à autre chose.

\section{CAMILLE}

Version $\mathbf{1}^{3}$
Les arbres
Les bruit de feuilles
Des grillage
Des buisson
Des sapins
Des maison
De l'herbe
Un teran de foot
Des bant
Des jeux
Des feuilles morts
Un grillage
Un bruit d'avion
Des voitures
Des bruit d'oiseau
Des fleurs
Le lycée
Un parking
Des mron
Des plantes
Des bruit de voiture
Des poubelles
L'hospital

\section{Version 2}

Je vois ${ }^{4}$; des arbres, des grillarge, des buisson, des sapins, des maisons, de l'herbe, un terrain de foot, des bants, des jeux, des feuilles mortes, un portaille, des voitures, des fleurs, le lycée, un parking, des marons, des plantes, des poubelles, l'hospital J'entends ; Les bruits d'oiseau, de feuilles mortes, des bruits d'avions

Version 3 (premier essai de mise en texte)

Je vois des arbres dans la courts.

J'entends les bruits de Feuilles mortes ${ }^{5}$ sur le sol.

fe vis des illage. ${ }^{6}$

Je regarde des buissons et à coté il s'y trouve des plantes

Dans le ciel j'écoute les bruits d'avion

f'ai remarqué des sapins à cotê de la maternelle.

Je suis aller à coté des grillage et j'ai vue des sapins.

fe vois des maisens à coté du parking.

J'ai été au portaille puis j'ai vue des maisons.

A cotê des arbres j'ai vue de l'herbe.

fe vis un te foot coté.

Sur le terrain de j'ai regarder des personnes jouer au foet.

$$
<\text { foot }>7 \quad<\text { ballon }>
$$

Sur la route les voitures font $\mathrm{du}$ bruit.

Dans la courts de récréations, les oiseaux font du bruit. $<$ chantes $>$

Version 4 : la copie de l'enfant

[Ponctuation à compléter. Les majuscules ont été respectées, c'est bien.

Il faudrait choisir entre raconter au présent et raconter au passé.

Attention aux répétitions.

\footnotetext{
${ }^{3}$ Notes prises dans la cour.

${ }^{4}$ En gras figurent les ajouts par rapport à la version précédente.

${ }^{5}$ En italiques figurent les suppressions par rapport à la version précédente.

${ }^{6}$ Les phrases barrées l'ont été par l'élève au moment de l'écriture.

${ }^{7}$ Les soufflets figurent les insertions après modification.
} 
Marie-Laure Elalouf et Carole Tisset

Tous les «je vois...j'entends... ont-ils été supprimés? $]^{8}$

Je vois des arbres dans la courts.

$<$ la court. La cours $>$

J'entends les bruits de feuilles mortes.

$<$ J'entends $><$ grinssement de feuilles mortes ; > $<$ grincement de feuilles mortes $>$

Je regarde des buissons et à coté il s'y trouve des plantes $<$ à côté $><$ se trouvesnt $><$ des petits sapins des arbustes. $>$

Dans le ciel j'écoute les bruits d'avion.

J'écoute les bruits d'avion dans le ciel.

Je suis aller à coté des grillage et j'ai vue des sapins. $<$ allée à côté des grillages j'ai aperçue $>$

$<$ vers $><$ les $><$ sont planter $><$ j'ai montrers des sapins $>$

J'ai été au portaille puis j'ai vue des maison.

$<$ au portailles $>\quad<$ des maisons $>$

Dans la cour du haut se trouve une salle de judo.

Sous le préau sont construit des toillettes. Mais nous n'avons pas de savon ni de papier. $<$ construits des toilettes $>$

\section{Version 4 recopiée}

Sur le plateau de foot sont tracées des lignes. Deux terrains un en haut de la cour, en bas.

$<$ Sur $>$

$$
<\text { sont placés }>
$$

Les côtés sont plantés plusieurs marronniers dont les feuilles mortes tombent comme les marrons. Le Midi ont sent la nourriture de la cantine.

Des voitures passent, des enfants crient à la récré dans la cour de la maternelle.

Dans la cour du haut se trouve une salle de judo.

Sous le préau sont construit des toillettes. Mais nous n'avons pas de savon ni de papier.

On remarque de nombreuses modifications apportées par l'élève à la suite des demandes de l'enseignante. Ce comportement est assez rare à l'école élémentaire pour le signaler, car les maîtres ne font guère retravailler les textes bien que les Instructions le leur recommandent depuis plus de 15 ans. Il ne s'agit pas chez eux d'un comportement dû à un maintien de la tradition mais du symptôme d'une vraie difficulté à faire reprendre un texte, difficulté liée au nombre d'élèves, donc au surcroît de travail, à l'impossibilité d'individualiser, mais aussi difficulté personnelle à percevoir «à chaud» ce qu'il convient de faire reprendre et de mobiliser les ressources linguistiques adéquates. Enfin, les enseignants de l'école élémentaire souhaitent généralement montrer des produits finis aboutis, ce qui est impossible sur des écrits.

La version 1 est une liste d'objets vus. Dans la version 2, l'élève fait suivre les verbes de perception de sa liste énumérative. Un texte prend forme dans la version 3 avec une continuité thématique sous la forme d'un je percevant, l'abandon de l'énumération pour des phrases simples, l'introduction de repères spatiaux.

\footnotetext{
${ }^{8}$ Les commentaires de l'enseignante apparaissent en italiques.
} 
L'anaphore constituée par la répétition de la formule «je + verbe de perception» donne une cohésion au texte. Mais ce texte ne correspond pas aux attentes de l'enseignante qui souhaite une progression à thèmes dérivés. Elle demande que les verbes de perception disparaissent. L'enfant s'y essaie en fin de texte dans les deux dernières lignes. Le je disparait. Les GN autrefois compléments sont devenus sujets syntaxiques mais au détriment de la continuité thématique car il n'y a plus de lien thématique entre je, la cour du baut et le préau.

Ces ruptures s'aggravent dans la dernière version 4 recopiée où l'enfant a respecté scrupuleusement la consigne de l'enseignant. Se succèdent alors des objets du discours sans lien les uns avec les autres. Pourtant apparaissent des indications spatiotemporelles: en haut, en bas, sur le côté, le midi qui auraient pu être mises en exergue pour aider à la mise en texte et créer une cohésion à partir d'un regard focalisateur externe organisant l'espace. L'énumération semble disparate parce que les constructions syntaxiques sont très variées, ce qui nuit également à la textualité. Enfin, la subjectivité réapparait sous la forme du nous et l'allusion au manque de savon et de papier dans les toilettes, qui est là comme une demande implicite d'intervention.

\subsection{Contournement mais assèchement}

La seconde série de textes montre comment un élève peut être amené à refuser, sans doute inconsciemment, certaines difficultés parce qu'elles lui paraissent insurmontables, parce qu'il ne comprend pas les raisons alléguées par l'enseignant pour effectuer des changements.

\section{GEOFFREY}

\section{Version 1 : prise de notes}

Sur le terrain de foot $<$ du haut $>$, je vois :

A ma droite il y a des arbres à coté du grillage qui mène à la cour du bas.

A ma gauche il y a le gymnase

Devant moi il y a les classes

J'entent les maternel qui joue.

Je sens la pollution à toute heure et à midi on sent se quont va manger

$$
\langle\text { qu'ont }>
$$

\section{Version 2 : premier essai de mise en texte}

Sur le terrain de foot (du haut) je vois :

a ma droite, il y a des arbres à coté du grillage qui,

$<\mathrm{A}>$

mène sépare la cour du haut et du bas.

a ma gauche il y a le gymnase.

$<\mathrm{A}>$

der

Devant moi il y a les classe. 
Marie-Laure Elalouf et Carole Tisset

J'entendt les maternel qui joue.

Je sens la pollution des voiture.

\section{Version 3 : deuxième essai de mise en texte}

Sur le terrain de foot (du haut) je vois que a ma droite ily a je regarde les des arbres qui sont à coté du grillage qui sépare la cour du baut et du bas.

Sur le terrain de foot (du haut), je vois que à ma droite, les a je regarde les arbres qui sont à coté du grillage <qui > séparant

$$
\text { séparent }
$$

les deux cour la cour du baut et du bas

$$
<\text { cours }>\text {. }
$$

A ma couche, c'est le gymase que vilì

A ma pauche, c'est le gymase. Que

de Devant moi, c'est les classes.

\section{Version 4 : copie}

[Ponctuation et majuscules ont été respectées, c'est bien.]

Sur le terrain de foot, à ma droite, les arbres, à coté du grillage qui séparent les deux cours.

A ma gauche, le gymnase est entreposé. <placé>.

\section{Version 5 : copie retravaillée}

[Recopier au stylo à bille noir en sautant une ligne.]

Dans la cour après la récréation du matin

Sur le terrain de foot, à ma droite, les arbres, à côté du grillage, séparent les deux cours.

A ma gauche, le gymnase est placé.

Dès la prise de notes, l'enfant écrit spontanément en utilisant des repères spatiaux (à ma droite, à ma gauche, devant moi). Dans la version 3, il essaie sans y parvenir de supprimer les verbes de perception, ce qui le bloque. Il produit des énoncés asyntaxiques constitués d'un GN expansé par une relative : des arbres qui sont... Agacé, il finit par barrer tous ses essais.

Dans la version 4, il tente de produire une phrase répondant aux exigences de l'enseignante, mais l'énoncé n'a plus de pivot verbal : A ma gauche, le gymnase sé $<$ placé $>$.

Dans la version finale, la cohésion textuelle est assurée par la position du sujet focalisateur autour duquel s'ordonne la description. Geoffrey, contrairement à Camille, a évité le piège de l'énumération d'objets grâce au maintien des indicateurs spatiaux. Mais son texte est réduit à deux phrases. De plus, la deuxième phrase illustre le conflit entre des contraintes lexicales avec l'inadéquation du verbe placer et des contraintes à la fois sémantiques et textuelles, le complément locatif du verbe « placer » ne supportant l'antéposition que dans certains cas. 
Thématisation, ruptures thématiques et réécriture dans les écrits scolaires

\subsection{Une solution : l'anaphore stylistique}

Le texte suivant propose une réponse très satisfaisante à la cohésion textuelle.

\section{PAULINE}

\section{Version 1 : prise de notes}

Je vois un terrain de foot. X2.

" " des flaques d'eau.

" " arbres.

$<$ sont plantés $>$

J'entend des cris d'enfants.

" des frotements de pied.

J'entend le vent.

Je vois des feuilles mortes dans le vent.

" " " voitures qui passent.

" " de la pelouse.

" " des bancs.

Je vois des un

\section{Version 3 : première copie \\ [Ponctuation et majuscules ont été respectées, c'est bien. \\ Toutes les phrases ont-elles un \\ verbe conjugué? \\ Attention aux répétitions. \\ Tous les "je vois...j'entends... » ont-ils été supprimés? ]}

Je vois des terrains de foot.

Des flaques d'eau mouilles le sol.

Des arbres qui sont platés

Des feuilles mortes sur la pelouse.

Des voitures passent devant l'école.

Des bancs verts sont installés.

J'entends des cris d'enfants.

J'entends le vent souffler.

J'entends les frottements

de pieds des autres.

\section{Version 2 : essai de mise en texte}

Je vois des terrains de foot.

Je vois Ddes flaques d'eau moulles le sol.

fe vis Ddes arbres qui [ratures illisibles].

Je vois Ddes feuilles mortes dans le vent sur la pelouse.

Je vois Ddes voitures sur un parking qui passent devant l'école.

fe vis de la pelouse.

fe Ddes bancs verts sont installés.

J'entends des cris d'enfants.

f'entends le vent souffler

$<$ J'entends. $>$

J'entends les frottements de pieds des autres.

$<$ J'entends $>$

\section{Version 3 : première copie corrigée}

fe vis des terrains de foot sont déssinés tracés.

Des flaques d'eau s'évaporent sur monilles le sol.

Des arbres quisont platés plantés sur

Des feuilles mortes sont tombées sur la pelouse.

Des voitures passent devant l'école.

Des bancs verts sont installés.

J'entends des cris d'enfants.

f'entends Je sent Je distingue le vent souffler dans les $<$ branches

d'>arbres.

f'entends Je discerne-les frottements de pieds des autres $<$ camarades $>$.

Les pieds de mes camarades frottent par terre. 
Marie-Laure Elalouf et Carole Tisset

\author{
Version 4 : deuxième copie \\ Des terrains de foot sont tracés sur le plateau. \\ Des flaques d'eau s'évaporent sur le sol. \\ Des arbres sont plantés dans la terre. \\ Des feuilles mortes sont tombées sur la pelouse. \\ Des voitures passent devant l'école. \\ Des bancs verts sont installés. \\ Des enfants crient. \\ Le vent souffle dans les branches d'arbres. \\ Les pieds de mes camarades frottent par terre.
}

Pauline a utilisé dès la prise de notes les verbes de perception. Dans la version 2, elle s'essaie à la suppression de ces verbes et tous les GN ne sont pas pourvus de verbes. Mais se fait jour un procédé littéraire amplement utilisé par Pérec: la nomenclature (Les choses, Pocket). La succession de thèmes certes est sans lien mais le parallélisme syntaxique, la conformité de la construction supplée ce manque : Des + $G N+$ expansion alternant régulièrement avec Des $+G N$ sans expansion (version 4 recopiée). L'anaphore, au sens stylistique fait office de liant. Cette anaphore est particulièrement sensible quand elle est interrompue aux deux dernières phrases avec des déterminants définis.

Le dispositif d'écriture observé en CM2 oblige les élèves à gérer simultanément trois des cinq catégories de contraintes recensées par Sylvie Plane (2003) : des contraintes d'ordre linguistique, des contraintes résultant de prescriptions imposées par la consigne et des contraintes imposées par le texte produit. La suppression des verbes de perception, exigée par l'enseignante, oblige les élèves à résoudre conjointement trois problèmes linguistiques de niveaux différents : syntaxique, puisque le complément du verbe de perception devient sujet de la phrase ; lexical, puisqu'il faut trouver un verbe compatible avec un nom non animé ; textuel, puisqu'à la progression à thème constant sur je, il faut substituer une progression à thèmes dérivés, cohérente avec l'hyperthème donné par la consigne. Il apparait que la gestion simultanée de ces trois problèmes excède les ressources cognitives et langagières des jeunes scripteurs qui satisfont aux exigences syntaxiques au détriment de la cohérence sémantique et textuelle. Le cas de Pauline est particulièrement intéressant : au lieu de renoncer à ses versions antérieures, elle en dégage une matrice qui concilie son propre projet d'écriture et les exigences de l'enseignante. C'est son exploitation du texte déjà produit, qui lui permet de dépasser de façon originale la difficulté qui bride les autres élèves.

Au total, les trois textes montrent des tentatives pour mobiliser des procédés linguistiques susceptibles d'assurer la cohérence de leur texte : la focalisation, l'anaphore stylistique et les connecteurs spatiaux. Mais ces procédés ne semblent pas contrôlés ni exploités. 


\section{Un dispositif d'écriture en fin de $5^{\mathrm{e}}$}

Les écrits analysés ici ont été produits à l'issue d'un ensemble de cinq séquences sur le Moyen Age, de mars à juin, ayant pour titre :

$$
\begin{aligned}
& \text { - connaître la littérature du Moyen Age } \\
& \text { - image et culture au Moyen Age } \\
& \text { - connaître la vie au Moyen Age } \\
& \text { - insérer une description, un portrait, un dialogue dans un récit } \\
& \text { - préparer l'écriture longue (un roman historique) }
\end{aligned}
$$

Nous comparerons deux écrits produits à la fin de cette période, dans des situations différentes : pour l'un, une évaluation sommative individuelle, pour l'autre, un écrit long produit par groupes (Elalouf \& Keraven, 2003).

\subsection{L'écriture à contrainte : quelles incidences sur la progression thématique?}

L'évaluation sommative est une écriture à contraintes. Le professeur demande à ses élèves, qui ont déjà lu des descriptions de monstres médiévaux, d'écrire selon la consigne suivante :

$$
\begin{aligned}
& \text { 1. Coloriage' } \\
& \text { 2. Description du « monstre » ( } 20 \text { à } 30 \text { lignes) } \\
& \text { Lui donner } 1 \text { nom commun et } 1 \text { nom propre. } \\
& \text { 3. Les « interdits »: } \\
& \text { 'Il y a' } \\
& \text { le verbe 'être' : pas plus de } 2 \text { fois } \\
& \text { 'petit', 'beau', 'grand', 'joli',... tous les mots banals }
\end{aligned}
$$

L'exigence de recherche lexicale, conforme aux canons de la stylistique scolaire, conduit les élèves à organiser leurs textes selon une progression qu'ils n'auraient pas nécessairement choisie, ce qui entraîne des perturbations, mais leur permet également d'expérimenter certains enchaînements.

\section{1. 1. L'interdit "il y a»: perturbations induites par la contrainte et gestion des ruptures thématiques}

\section{RACHID}

Un monstre naturelle au millieu de belles fleurs (rose, tulipe, fougère, raisin... etc). il reste toujours avec ses fleur.

\footnotetext{
${ }^{9}$ Deux dessins de monstre au choix ont été distribués. Voir p. 111 la reproduction du dessin choisi par les élèves dont les textes sont présentés.
} 
Il ce nome florisare. Sur son museau on voit une corne qui lui sert à déchicter n'importe quoi (le gibier) et sa crinière éblonissante, sur sa tête une flame l'éclaire la nuit. Sur son dos se traine une carapace aux couleurs aveuglantes $I l$ mesure $1 \mathrm{~m}$ de long et $80 \mathrm{~cm}$ de large, florisare pèse $120 \mathrm{~kg}$. au bout de sa queue vert tombe une fourche dont trois pic qui servent à griffé ${ }^{10}$.

La griffe sur sa jambe lui sert à acracher le sol pour courrir plus vite.

Dans le fond florisare se montre méchant et très agréssife si on l'énerve.

Mais si on le laisse tranquile il ne fera de mal à perssonne il veut seulement rester seul avec ses fleurs. La creatures s'amuse beaucoup avec ses plantes.

On peut supposer que Rachid commence son texte par une phrase non verbale pour éviter l'emploi du présentatif. Mais elle ne constitue pas pour autant un titre et la progression linéaire qui s'établit avec la phrase verbale suivante pose problème, alors qu'elle aurait été naturelle après une phrase à présentatif. Mais ensuite, se développe une progression à thèmes dérivés, avec quatre phrases qui proposent des variations autour d'un même schéma :

$$
\begin{aligned}
& \text { - groupe prépositionnel en position thématique : 'sur }+ \text { déterminant possessif } \\
& + \text { partie du corps' } \\
& \text { - sujet - verbe - complément : } \\
& \circ \quad \text { on voit une corne : l'élément nouveau est en position rhématique, } \\
& \text { complément d'un verbe de perception; } \\
& \circ \quad \text { une flamme l'éclaire : l'élément nouveau correspond au « reste du } \\
& \text { thème » : il est sujet de la phrase; } \\
& \circ \quad \begin{array}{l}
\text { se traine une carapace, tombe une fourche : l'inversion du sujet permet à } \\
\text { celui-ci d'être en position rhématique. }
\end{array}
\end{aligned}
$$

Seule la coordination des deux groupes nominaux une corne (...) et sa crinière éblouissante maintient une discordance, du fait du statut informationnel différent des deux syntagmes coordonnés.

\section{1. 2. L'interdit "est»: perturbations induites par la contrainte et} gestion des ruptures thématiques

\section{MÉLANIE}

Version définitive

Galopa Fleuri, "la chose étrange », [rempli, -i souligné deux fois par P] de couleurs splendides et de ravissantes fleurs comme le montre son prénom [phrase incomplète]. Sa tête semblable à celle d'un cheval avec ses yeux bleus luisants, une crinière et son poil brun $[i d]$.

\footnotetext{
${ }^{10}$ Les perturbations dont on pense qu'elles sont liées à l'observation de la consigne sont signalées en italiques gras. Les phrases où s'instaure une progression à thèmes dérivés avec antéposition d'un groupe prépositionnel et introduction d'un élément nouveau dans la suite de la phrase sont soulignées. Quand l'élément nouveau est en position thématique, il est en caractères gras, droits.
} 
Son corps mince et violet qui ressemble à un magnifique artichaut multicolore [id]. Sa patte, un peu plus charnue que la tête, d'où sort une griffe presque interminable $[i d]$. A l'extrémité du corps, une queue épaisse et violette puis au bout une tulipe rouge finie cette queue $[i d]$.

«La chose étrange » mange une carotte orange et verte. De son corps [sort -t souligné par $P]$ des tiges vertes avec des fleurs comme finitions.

Galopa fleuri, mélange d'un cheval pour la tête, d'un artichaut pour le corps, de la patte d'un dragon et de la patte d'un dinosaure.

Ses fleurs de différentes formes et de différentes couleurs comme le rose, le bleu, le rouge, le vert décorent cet remarquable « chose étrange ». Galopa Fleuri écrase une tulipe jaune avec sa patte en souriant et en mangeant.

Galopa Fleuri, très sympatique avec tout le monde malgré son aspect physique monstrueux et étrange [id].

\section{Commentaire du professeur}

Quand le sujet grammatical de la phrase est un peu long, tu perds de vue qu'il faut un prédicat $(G V=V+$ complément / attribut)

Dans le texte de Mélanie, les phrases nominales prédominent, sans que l'on sache s'il s'agit d'un choix délibéré de l'élève ou d'une difficulté résultant de la consigne. L'alternance de phrases verbales et non verbales étant liée à la présence ou non d'un verbe dynamique, on penche pour la seconde hypothèse. Le diagnostic du professeur est que l'élève, attachée à inclure des éléments descriptifs dans des groupes nominaux expansés, perd de vue la structure sujet-prédicat. C'est possible pour les phrases 3 et 4 . Pourtant, dans d'autres phrases non verbales, une répartition de l'information en thème et rhème est observable (ex : phrase 1,2$)$. On peut avancer l'idée que ces constructions résultent plutôt de l'interdit relatif au verbe être. Toutefois, comme chez Rachid, on trouve une progression à thèmes dérivés dans laquelle les éléments nouveaux sont en position rhématique (phrases soulignées).

\section{1. 3. Des ruptures thématiques mettant en relief un détail descriptif}

\section{PRISCILLA}

\section{Version définitive}

Le rhinocéros atteind une queue très longue comme celle d'un lion. Une jambe de kangourou toute poilue avec une griffe bien aiguisée. "Lucifer» comprend une tête de cheval coloré. Il a des écailles multicolore vives qui reflette au soleil. Sa corne bleu, large et poilu a des traits commun avec un couteau. Son regard orange fait peur quand-il semble nous regarder et semble garder un terrible secret. Il porte une barbichette violette assez minime ressemblant à celle d'une chèvre.

Des branches et des fleurs fantastique de toute les couleurs sortent de son magnifique corps mélangé de toute sorte d'animaux assez brute. Le 
Marie-Laure Elalouf et Carole Tisset

rhinocéros nous sourit d'un air sévère, énervé et agacé mais pas sympatique du tout. Sa crinière couleur orange-rose est bien propre et coiffé Son oreille ample et haute ressemble étrangement à celle d'un chien. Ses sourcils sont épais et d'un noir ténébreux. On entend «Lucifer» respiré de loin car ses narines atteignent une profondeur et une largeur immenses.

Le texte de Priscilla comporte deux paragraphes symétriques, qui s'ouvrent selon une progression à thème constant, faisant place au milieu de chaque paragraphe à une progression à thèmes dérivés. Malgré la perturbation liée à l'interdit il y a (phrase 2) et celle liée à l'interdit, implicite, des répétitions, qui conduit vraisemblablement l'élève à préférer au verbe avoir atteindre (phrase 1) et comprendre (phrase 3), cette symétrie, soulignée par les désignations du monstre (le rbinocéros ... Lucifer ..., il, le rbinocéros ... Lucifer) rend possible la rupture thématique en début de second paragraphe, qui met en relief le caractère proprement monstrueux de l'alliance du végétal et de l'animal.

\section{1. 4. Des brouillon productifs}

\section{SANDRINE}

\section{Brouillon}

Ce monstre s'appelle Dragon-fleur. Il est constitué d'une tête de cheval, d'un corps de dragon et de deux bras en forme de plusieurs tiges qui se terminent par des fleurs. La mystérieuse créature attire peut attirer des gens grâce à ses multiples couleurs. Ses yeux aux contours violets expriment la menace mais à l'intérieur cette couleur bleu ciel adoucit son regard. La créature comporte une peau couleur marron clair et une crinière d'un jaune citron que l'on voit de loin. Sa tête se finit par Une barbichette noir profond de bouc, fini son menton. Au dessus de son nez museau une corne grise de rhinocéros, plantée là comme par miracle lui permet de se défendre. Son corpulent corps de dragon d'un vert émeraude avee a une queue dont la fin rougeâtre, ressemble à du sang. Une fleur qui mélange le rose foncé et le violet dissimule une partie du corps de Dragon-fleur. ${ }^{*}$ Un crochet rougeatre comme la queue de la créature, ressort de son unique jambe. Ses bras bleu nuit, se dispersent en des tiges finissant par des fleurs différentes sortes de fleurs orange, violet, rose, rose très puissant, bleu, violet, orange. Une des fleurs du monstre mystérieux dragon peut se comparer à une main d'humain.

*La partie inférieur de cet être se termine par une tige possédant des fleurs, elle aussi.

Une place importante étant accordée au travail du brouillon dans cette classe, on peut se demander si la consigne proposée conduit les élèves à envisager différents types de progression. C'est ce que l'on observe dans le brouillon de Sandrine, qui renonce à un enchaînement thématique pour introduire une rupture ("Sa tête se finit par Une barbichette noir profond de bouc, fini son menton») et insère un ajout pour maintenir une alternance entre rupture thématique et retour à la progression initiale. On note en outre qu'elle substitue le nom propre, puis une désignation nouvelle à une anaphore générique (" Dragon-fleur; du mystérieux dragon»). 
Thématisation, ruptures thématiques et réerriture dans les écrits scolaires

\section{MAXIME}

Brouillon 1

Le monstre s'apellait chevalartigon et ce nom lui allait bien car sa tête ressemblait à une tête de cheval, son corps avait la forme d'une plante, l'artichaut, sa jambe et sa queue parraissait être des membres de dragon.

Sa tête était marron clair il avait sur son museau une immense corne pour se défendre. Sous sa bouche un bouc jaune pendait. Ses yeux sombres et terrifiant intriguait ses adversaires. Deux immense oreilles bondissait au dessus de son crane, en dessous se trouvait une magnifique crinière couleur or...

Son buste faisait penser à un artichaut, son corps détenait de sublime couleur, du vert, du rouge et du jaune. De son torse sortait des lianes au bout des fleurs, des feuilles mais aussi une main et une boule. Avec son unique patte il pouvait tuer ses adversaires à l'aide d'une griffe pointu

Brouillon 2

Le monstre s'apellait chevalarpoigon et ce nom lui va bien car sa tête ressemblait à une tête de cheval. Le haut de son corps avait la forme d'une plante et le bas de poisson car il possède des nombreuse écailles. Sa queue et sa jambe semble être des membres de dragon.

Sa tête était marron clair en dessous de ses dents rouge de sang pend un gigantesque bouc de couleur jaune. Sur son museau une immense corne lui sert à se défendre, deux immenses oreilles se tiennent sur sa tête. Plus bas une splendide magnifique crinière couleur or. Ses yeux sombres et terrifiant intriguent ses adversaire.

Son buste faisait penser à un artichaut grace a ses feuilles qui détiennent de magnifiques couleurs vert, rouge et jaune. De la partie haute du torse sortent des lianes avec au-bout des feuilles et fleurs mais aussi une main, une boule et une sorte d'oursin. La partie du bas possède des écailles qui lui permet d'aller dans l'eau.

A l'aide de son unique patte il peut tuer ses ennemis avec une griffe acérée. Sa patte et sa queue a une couleur bleu. Au bout de cette queue une flamme de couleur orange éclairé les endroits sombres

Chez Maxime, un mode de progression s'instaure dans le premier état du texte qui est développé dans le second, avec une alternance entre des phrases dont le thème propre est sujet (sa tête, son buste) et d'autres dont le thème propre est un groupe prépositionnel antéposé (en dessous de ses dents rouge de sang, sur son museau, de la partie haute $d u$ torse), les deux éléments nouveaux en position thématique se trouvant dans deux phrases successives dont la symétrie est renforcée.

Il est difficile de savoir si ces évolutions résultent de calculs ou de tâtonnements. Dans la mesure où aucune notion de progression de texte n'a été explicitement introduite, on penche pour la seconde solution, en faisant l'hypothèse que le dispositif d'écriture a permis aux élèves d'explorer des agencements textuels auxquels ils ont donné une cohérence. Comparés aux textes de CM2, ceux de $5^{\mathrm{e}}$ sont moins perturbés par les contraintes syntaxiques et lexicales induites par les «interdits », sans doute parce que les ressources langagières d'élèves plus âgés sont plus grandes - elles ont d'ailleurs été développées par un travail spécifique sur le vocabulaire, la caractérisation et la comparaison (Elalouf, 2005), mais aussi parce que les élèves se sont approprié le référent imaginaire décrit en le coloriant, puis en lui donnant un nom. 


\section{2. Des bribes de descriptions, écrits au préalable, à leur insertion dans le roman : ruptures thématiques ignorées, contournées, assumées}

L'autre situation d'écriture observée est la rédaction, par groupes de 3 ou 4 élèves d'un roman historique, lieu de réinvestissement des recherches documentaires et lexicales sur le Moyen Age. Là, le professeur pose non pas des interdits mais des passages obligés : le héros du roman sera un ménestrel, chaque personnage sera décrit, selon son importance, dans un portrait qui pourra se faire en plusieurs temps, de même que les lieux ou scènes suivants : un château fort, un tournoi, une attaque du château fort, une fête villageoise, un déplacement dans la campagne du personnage principal, un festin dans la grande salle du château. Et pour prolonger la réflexion menée en lecture sur les fonctions de la description, l'enseignante demande aux élèves, qui ont déjà construit un synopsis, de rédiger des descriptions et de les justifier avant de commencer l'écriture longue proprement dite. Lors de la rédaction du roman historique, la question de l'intégration de ces descriptions se trouvera posée : comment les insérer? Faut-il apporter des modifications pour que cette insertion soit pertinente et n'apparaisse pas comme un corps étranger dans la progression du texte? Selon les groupes d'élèves, les choix opérés sont différents.

\section{2. 1. Collage et ruptures syntaxiques (roman 2)}

\section{Synopsis}

Le portrait du héros : Un ménestrel

Identité : Martin le franc

Statut social : Le père de Martin était un musicien très connu. A sa mort Martin n'avait que 8 ans et il dut suivre l'exemple de son père pour survivre.

Traits physiques : Moyen de taille, les yeux marrons, les doigts très fins, le regard vif et fort

Profession, goûts : Fils unique, il c'est lancé dans une carrière de ménestrel.

Comportement : intelligent, courageux

\section{Bribes}

Un méchant seigneur (chef des bandits) : homme riche, qui a soif de pouvoir, des yeux vicieux, habillé de noir, il a déjà tué plusieurs personne

\section{Roman}

Pendant le festin, un ménestrel s'appelant Martin, moyen de taille, les yeux marrons, les doigts très fins, le regard vif et fort, une petite bouche, les cheveux longs et bruns, de grandes jambes s'apprêtait à entrer en scène quand soudain l'olifant se fit entendre car des bandits et leur chef qui est un homme riche, assoiffé de pouvoir, des yeux vicieux, habillé de noir, très réservé, il a déjà tué plusieurs personnes, attaquaient le château ${ }^{11}$.

Le groupe 2 s'en tient à un collage qui a pour effet de disjoindre du verbe la tête du groupe nominal sujet, par l'insertion de constructions détachées de formes variées,

\footnotetext{
${ }^{11}$ Les ajouts entre l'état n et l'état $\mathrm{n}+1$ sont indiqués en gras, les modifications en italiques gras.
} 
sans ordre logique apparent et même d'une phrase qui se caractériserait à l'oral par une rupture intonative vers le bas ${ }^{12}$ : «il a déjà tué plusieurs personnes». Ces élèves produisent ainsi une phrase beaucoup plus développée que dans leurs autres écrits, mais ne perçoivent pas la nécessité de la réécrire.

\section{2. 2. Bouturage et ruptures thématiques (roman 3)}

\section{Bribes}

\section{Description du chevalier}

Constantien appelé Constentien le chevalier ne se sépare jamais de son cheval blanc $\grave{a}$ la longue crinière. Constantien est blond au cheveu mi-long et aux yeux verts. Il est maigre, silhouette fine, de taille moyenne, il s'babille souvent d'une tunique en laine grossière. Il n'a presque jamais peur sauf des rats il est adroit et généreux.

\section{Roman, état 1}

A Fontainebleau, en l'an 1042, dans les parcs du château, Constentien le chevalier et Picasnon-le-puant, assis sous un pommier, virent tout à coup un pichet dans la fontaine. Constentien ne se sépare jamais de son cheval blanc avec une longue crinière.

Il est blond aux cheveux mi-longs et aux yeux verts. Celui-ci est maigre, avec une silhouette fine, de taille moyenne. Il s'habille souvent d'une tunique en laine grossière. Hn n'a llats est adroit et généreux. Picasnon-lepuant est petit et gros, puant, plein de crasse avec des habits vert foncé et déchirés, il ne s'est jamais lavé. C'est l'écuyer de Constentien, il a des cheveux bruns et courts, des yeux marrons, un nez crochu, de grosses lèvres rouges, un menton prognathe, il est bossu il a des cheveux courts, il est trapu et il a des habits verts fonés.

Le groupe 3 part de la description d'un personnage, selon une progression à thème constant. Au moment de l'écriture du roman s'impose à eux l'idée d'un couple de deux personnages antithétiques. Un détail bas est supprimé du portrait du chevalier (Il n'a presque jamais peur sauf des rats) tandis que celui de Picasnon multiplie les épithètes péjoratives. Le fragment descriptif initial fonctionne ici comme une bouture qui génère le texte. Mais la gestion de cette opposition sur le plan thématique est plus délicate : comme le démonstratif celui-ci n'annonce pas un contraste avec celui-là, il perturbe la première progression à thème constant (portrait de Constentien). Par ailleurs, la phrase c'est l'écuyer de Constentien interrompt la seconde progression à thème constant (portrait de Picasnon), alors que l'information aurait pu figurer sous forme de prédicat second.

12 Morel, M.-A. ; Danon-Boileau, L. (1998) : Grammaire de l'intonation. Paris, Ophrys. 
Marie-Laure Elalouf et Carole Tisset

2.2.3. L'intégration de fragments descriptifs : stratégie de contournement ? (roman 1)

\section{Bribes}

Château : grand, entour d'une profonde douve, 4 tours, un pont levi, une herse, et puis les portes en bois du donjon, une grande salle pour les festins. avec il y a une grande table arrondi avec de grande nappe blanche ou sont posés les couverts. Des chandeliers sont suspendus au plafond et d'autre posés sur la table. Des têtes d'animaux accroché au mur qui provenait de la chasse étaient accroché au sur les murs à côté une porte sépare la salle des festins de la cuisine ou le maitre queux prépare les repas dans de vaste fournaux. Au dessus de la salle des festins se trouvait les chambres dont les chambres du roi. A dextre ( $\neq$ senestre) se trouvait la chambre d'ami ou dormai le menestrel. Sa chambre était étroite avec un petit lit et une fenêtre qui éclairait la pièce.

\section{Roman, état 1}

Lorsque le jeune ménestrel poussa la porte du château et vit les personnes en larmes.

\section{Roman, état 2}

Lorsque le jeune ménestrel poussa la porte du château. Ce château est grand, entouré d'une profonde douve Une toure est au quatre coins du château, un pont levis puis une grande herse et d'immense porte en bois qui sont à l'entrée du donjon. Le ménestrel vit les personnes en larme.

\section{Roman, état définitif}

Un jour, Roland, le jeune ménestrel, poussa la porte du château, découvrit les profondes douves, le pont-levis et la grande herse. En entrant, il s'aperçut que tout le monde pleurait.

La stratégie du groupe 1 est plus habile, mais on peut se demander si elle ne permet pas de contourner une difficulté plutôt que de l'affronter. La première description du château suit une progression thématique peu lisible : énumération à valeur rhématique, présentatif, ruptures thématiques, progression à thèmes dérivés puis linéaire. Le premier état du roman n'intègre pas cette description. Le second en réintroduit certains éléments par une progression linéaire, suivie d'une rupture thématique que l'emploi distributif du déterminant un (une tour est aux quatre coins $d u$ château) puis l'ellipse du verbe être (un pont levis, puis une grande herse et d'immense porte en bois) et son rétablissement dans une relative (qui sont à l'entrée du château) rendent difficilement acceptable. L'évitement du présentatif s'avère coûteux sur le plan syntaxique alors qu'on aurait eu sans difficulté (Il y a une tour aux quatre coins du château, un pont-levis, une grande herse et d'immenses portes en bois à l'entrée du donjon). Dans l'état définitif, les éléments descriptifs apparaissent après des verbes d'action ou de perception dont le sujet est le jeune ménestrel, ce qui présente l'avantage d'instaurer un point de vue, mais évite de modifier la progression à thème constant centrée sur le personnage principal. 


\section{2. 4. L'intégration avec changement registre et modification de la progression thématique (roman 4) \\ Bribes}

Le festin dans la grande salle du château : Tous les nobles et chevaliers s'y retrouvent pour manger et parler en riant. Il y a des acrobates, des ménestrel et des musiciens. Les nobles et chevaliers rigolent s'amusent beaucoup. Le repas est très varié et bien garni, Ils mangent tous comme des cochons.

\section{Roman, état définitif}

Quelques minutes après, pour le festin qui se déroule dans la grande salle, mille cochons, trois mille faisans et sept mille lapins ont été commandés. Les serviteurs ont dressé des tables sur des tréteaux. Il y a plein de mets et de vins épicés. Jehanne et Roland sont assis au milieu des convives et admirent les spectacles des jongleurs et des musiciens. Tout à coup elle se lève, prend sa harpe, s'installe au milieu de la grande salle et joue. Encore une fois, le duc, Roland et les convives sont époustouflés par sa musique. Roland se dit au fond de lui qu'elle est si belle quand elle joue.

Le seul groupe ayant véritablement transformé les descriptions initiales est aussi celui qui produit également le texte le plus long et le plus travaillé. Dans la première description, le pronom anaphorique $y$ assure une continuité thématique et permet l'introduction progressive des personnages dans la salle du festin: les nobles et chevaliers, des acrobates, des ménestrels et des musiciens, ce qui rend possible par la suite la reprise des thèmes déjà introduits : les nobles et chevaliers, le repas. La description du festin adopte ici un registre bas (rigolent, mangent comme des cochons). Dans le roman, la description intervient en conclusion du récit, pour clore la scène des épousailles de Jehanne, le ménestrel qui se révèle être une jeune fille et Roland, le chevalier qu'elle a sauvé par amour. Les termes péjoratifs sont supprimés au profit d'une expression hyperbolique ; la progression est également modifiée, de façon que le repas soit le cadre prestation de Jehanne, qui prend une dimension symbolique.

Le second dispositif pratiqué dans cette classe de $5^{\mathrm{e}}$ comporte des points communs avec celui de CM2 : dans les deux cas, l'enseignante impose un ordre à la genèse de l'écriture, allant de la liste au texte. Les listes relèvent de genres différents : notes prises dans la cour en CM2, synopsis dont le cadre est fourni par l'enseignante en $5^{\mathrm{e}}$, premières descriptions qui, par leur forme énumérative, se démarquent à peine des synopsis. La différence fondamentale est que les notes prises en CM2 ne s'inscrivent pas pour les élèves dans un projet précis alors que la rédaction du synopsis est fortement orientée vers l'écriture longue. Passer de la liste au texte exige une linéarisation des énoncés et confronte les élèves à des choix de progression différents selon qu'ils ont à écrire un texte descriptif autonome ou subordonné à un texte narratif.

On peut à la lumière de ces deux exemples s'interroger sur la façon de confronter progressivement les élèves à la question des ruptures thématiques. La comparaison entre les textes produits en $\mathrm{CM} 2$ et en $5^{\mathrm{e}}$ apporte une réponse empirique à la question posée par B. Combettes (1986) dans son article sur l'introduction et la 
reprise des éléments d'un texte : «il conviendrait d'ailleurs de s’interroger sur la façon dont l'acquisition d'une compétence textuelle dans ce domaine est en rapport avec la distinction premier plan/ second plan.; on peut en effet penser que l'introduction d'éléments nouveaux ne s'effectue pas d'une manière identique dans des textes où le premier plan domine (en schématisant: les textes narratifs) et dans les textes où le second plan l'emporte (toujours en schématisant: les textes descriptifs); le texte narratif entraînera beaucoup plus facilement le «passage » par le personnage ou le locuteur et se prêtera moins aux autres types d'introducteurs; le texte descriptif soulèvera évidemment d'autres problèmes : la question se pose de savoir à quel moment, dans le développement de la compétence textuelle, le second plan ne se trouve plus «subordonné » au narratif, devient autonome et structuré comme un type de texte particulier, avec ses contraintes propres». On a pu observer combien l'interdiction de "passer par le locuteur», avec la suppression des verbes de perception, était source de dysfonctionnements en CM2. En 5e, l'écriture d'un texte descriptif autonome devient possible, associée à des lectures et à une réflexion sur les fonctions de la description, et les contraintes en sont d'autant mieux perçues quand il s'agit d'insérer la description dans un récit.

\section{Conclusion}

L'étude de ces deux corpus montre que les élèves, confrontés à des tâches d'écriture les obligeant à user de progressions thématiques auxquelles ils n'auraient pas spontanément recours, sont amenés à explorer des enchaînements variés mais que tous n'en tirent pas un égal profit. D'où la question : comment faire progresser ceux dont les textes semblent plus perturbés qu'améliorés par l'observation des contraintes? Quel type d'intervention didactique peut les conduire à une réécriture plus satisfaisante?

Nous ne sommes pas persuadées qu'à ce niveau d'enseignement, l'introduction des notions de thème et de rhème ou propos soient souhaitables. Distinguer le niveau de l'organisation syntaxique et celui de la perspective fonctionnelle suppose un niveau d'abstraction qui nous semble hors de portée d'élèves de cet âge (10-14 ans). De fait, les quelques grammaires scolaires qui introduisent ces notions dans ces classes les rabattent sur les fonctions syntaxiques en identifiant le sujet au thème, ce qui est source de confusion ${ }^{13}$. Il semblerait plus pertinent d'attendre la fin de la scolarité obligatoire pour reprendre sous un autre angle l'articulation entre organisation syntaxique et apport d'information.

En revanche, l'intervention didactique peut, sans métalangage particulier, sensibiliser à la variété des enchaînements possibles et aux contraintes qui pèsent sur eux. La suite de texte, exercice très pratiqué à l'école et au collège, permet d'aborder les questions d'enchaînement avec le texte-source (Corblin, 2006). Au moment de la réécriture, on peut proposer aux élèves de choisir entre plusieurs réécritures mettant

\footnotetext{
13 Ainsi, le sujet est souvent défini comme «ce dont on parle ». Inversement le thème peut être défini comme le point de départ de l'énoncé avec uniquement des exemples de sujet (STISSI, D. \& al. Grammaire pour lire et écrire, 6e, Delagrave, 1996).
} 
en jeu différents types de progression ou de ruptures en demandant d'expliquer leur choix ; on peut aussi attirer leur attention sur de possibles ambiguités en donnant plusieurs gloses d'un même enchaînement.

Mais cela suppose que l'enseignant y ait été préparé, c'est-à-dire qu'il soit capable, non seulement d'identifier des progressions types, ce à quoi se limite trop souvent la formation, mais aussi d'interpréter les changements de progression et les ruptures, de les mettre en relation avec d'autres phénomènes d'ordre syntaxique, lexical, énonciatif et d'interroger les textes d'élèves à la lumière de ces connaissances, en identifiant les niveaux d'analyse sur lesquels intervenir pour améliorer la cohésion de ces textes. L'enseignant a donc besoin de recul pour objectiver les textes que les enfants lui présentent, de connaissances linguistiques et de temps pour les analyser, de repères en didactique de la production d'écrits pour éviter l'effet du modèle implicite, trois conditions nécessaires mais difficiles à réunir dans l'enseignement du premier comme du second degré.

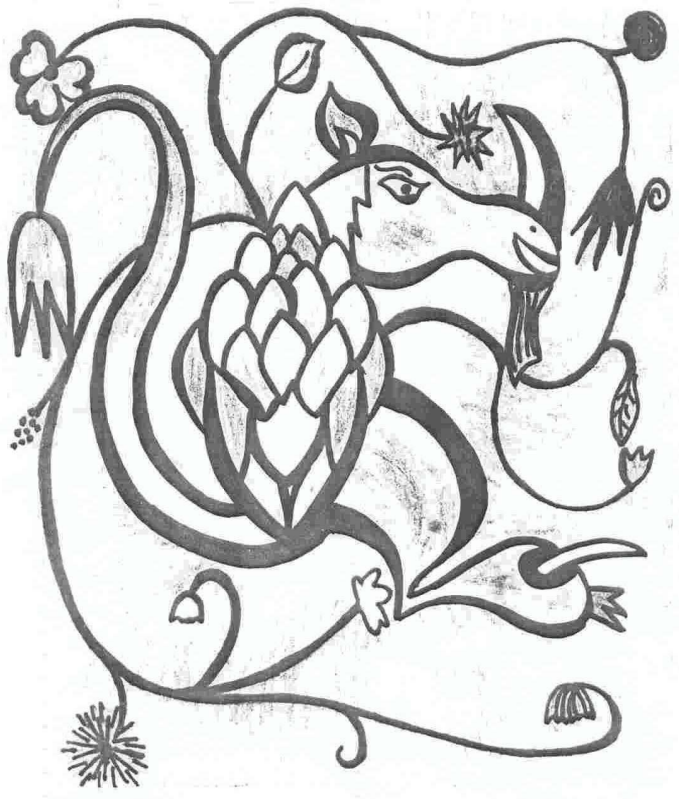


Marie-Laure Elalouf et Carole Tisset

\section{BIBLIOGRAPHIE}

ADAM, J.-M., 1984, Le Récit, Presses universitaires de France, Paris.

ANIS, J. \& BORE, C. (ed), 2004, «Théories de l'écriture et pratiques scolaires », Linx , 51, Paris.

Bessonnat, D. (ed.), 2000, « La réécriture », Pratiques, 105-106, Metz.

BronCKART, J.-P., 1985, Le fonctionnement des discours, Delachaux-Niestlé, Neuchâtel.

Bautier, E., Bucheton, D., 1995, «L'écriture : qu'est-ce qui s'apprend? Qu'est-ce qui s'enseigne, qu'est-ce qui est déjà là ? » in Le Français aujourd'bui, 111, Paris, pp. 26-35

Bonnet, C., Corblin, C., Elalouf, M.-L., 1998, Les procédés d'écriture chez les élèves de 10 à 13 ans, un stade de développement, CVRP, Lausanne.

Charolles, M., 1989, "Problèmes de la cohérence textuelle », in Pour une didactique de l'écriture, Pratiques et Centre d'Analyse Syntaxique de l'Université de Metz (Eds), Metz, pp. 49-83.

CombetTes, B., 1978, "Thématisation et progression thématique dans les récits d'enfants », Langue française, 38, Larousse, Paris, pp. 74-82.

Combettes, B., 1983, Pour une linguistique textuelle : la progression thématique, De Boeck-Duculot, Paris-Bruxelles.

Combettes, B., 1986, «Introduction et reprise des éléments d'un texte », Pratiques, 49, Metz, pp. 69-84.

COMBETTES, B., TOMASSONE, R., 1991, Le texte informatif, aspects linguistiques, Editions UniversitairesDe Boeck, Bruxelles.

Corblin, C., 2006, «Evaluations et commentaires en narration : effets sur des écrits du cycle 3 », Repères, 33, INRP, Lyon, pp. 105-119.

Danes, F., (ed.),1974, Papers on functional Sentence perspective. Prague, La Haye.

David, J., PLANE, S., 1996, L'apprentissage de l'écriture, de l'école au collège, PUF, Paris.

DE WeCK, G., 1991, La cohésion dans les textes d'enfants; étude du développement des processus anaphoriques. Neuchâtel, Paris, Delachaux \& Niestlé.

Elalouf, M-L, Keraven, J. (2003) : «Ecrits exploratoires, écrits à contraintes. Quelle complémentarité en classe de $5^{e}$ ?», Littératures et Langages, 1, Ecritures. CRDP de l'Académie de Versailles, pp. 149-156.

Elalouf, M.-L. (dir.) (2005) : Ecrire entre 10 et 14 ans, un corpus, des analyses et des repères pour la formation. CRDP de Versailles.

FABRE-Cols, C., 2000, Apprendre à lire des textes d'enfants, De Boeck-Duculot, Bruxelles.

FAYOL, M., GOMBERT, J. -M., 1987, «Le retour de l'auteur sur son texte : bilan provisoire des recherches en psycholinguistique », Repères, 73, INRP, Paris.

Garcia-Debanc, C., 1986, «Processus rédactionnel et pédagogie de l'écriture », Pratiques, 49 , pp. 23-49. 
Garcia-Debanc, C., Confais, J. -P., Grandaty, M., 2001, Quelles grammaire enseigner à l'école et au collège? Discours, genres, texte, phrase, Paris, Delagrave-CRDP Midi Pyrénées.

Halte, J.-F., Petitjean, A., Plane, S. (ed.), 2002, «L'écriture et son apprentissage. Questions pour la didactique, apports de la didactique », Pratiques, 115-116, Metz.

Mathesius, V. , 1975, A functional analysis of present day English on a general linguistic basis. La HayeParis.

M.E.N., 2002, Qu'apprend-on à l'école élémentaire? Les nouveaux programmes, C.N.D.P./ XO éditions, Paris.

Plane, S., 2003, «Stratégies de réécriture et gestion des contraintes d'écriture par des élèves de l'école élémentaire : ce que nous apprennent des écrits d'enfants sur l'écriture ». Rome Rivista Italiana de Psicolinguistica Applicata, anno III/1, pp.57-77.

Plane, S., 2002-2003, Repères, 26-27, 2002-2003, «L'écriture et son apprentissage à l'école élémentaire », INRP, Paris.

REUTER, Y., 2000, La description, des théories à l'enseignement-apprentissage, ESF, Paris.

Reichler-Beguelin, M.-J., Devernaud, M. \& Jespersen, J. , 1988, a, Écrire en français, cohésion textuelle et apprentissage de l'expression écrite. Neuchâtel, Delachaux \& Niestlé.

SCHNEUwLy, B., 1997, «Les genres scolaires. Des pratiques scolaires aux objets d'enseignement». Repères, 15, pp. 27-40. 
